\title{
MELT INCLUSIONS:
}

\section{PRINCIPAIS CARACTERÍSTICAS E TÉCNICAS DE ESTUDO}

\author{
Lucas Eustáquio Dias Amorim ${ }^{1 *}$, Monica Elizetti Freitas ${ }^{1}$, Francisco Javier Rios ${ }^{1}$, \\ Tatiana Aparecida Fernandes de Lima ${ }^{1,2}$
}

\begin{abstract}
1- Laboratório de Inclusões Fluidas e Metalogênese, SETEM- CDTN-CNEN-UFMG, Av. Antônio Carlos, 6.627 - Campus da UFMG, 31270-901 caixa postal 941, Belo Horizonte, MG; ledamorim@cdtn.br*, javier@cdtn.br, monicaef@gmail.com

2- Programa de Pós-graduação em Geologia da UFMG, Av. Antônio Carlos, 6.627, Instituto de Geociências, Campus da UFMG. $31270-901$. Belo Horizonte, MG; tatiaflima@gmail.com
\end{abstract}

Recebido em 15 de outubro de 2012; aceito em 25 de fevereiro de 2012

RESUMO: Melt Inclusions (MI) são pequenas porções do fundido magmático formador da rocha que durante o processo de cristalização ficaram aprisionadas em irregularidades dos minerais. Suas formas são muito variadas e refletem sua maturação após aprisionamento. A distribuição das MI depende do mineral hospedeiro: pode ser irregular (na olivina e clinopiroxênio), acompanhar as linhas de crescimento (no plagioclásio) ou estar associada a irregularidades no crescimento dos cristais (como no quartzo). Se as MI se comportaram como um sistema fechado, ficando protegidas de perdas ou alterações durante a ascensão do magma até chegar à superfície, elas constituem uma ferramenta poderosa para a reconstituição das características do magma no momento do seu aprisionamento. Por esta razão, as MI têm sido usadas em estudos petrológicos e petrogenéticos de sistemas magmáticos, ou ainda para a compreensão dos processos responsáveis pela mineralização em depósitos magmáticos hidrotermais. O estudo de MI permite a obtenção, de forma direta, de dados composicionais e físico-químicos do fundido magmático no estágio de cristalização do mineral hospedeiro. Com o objetivo de difundir essa técnica apresentam-se nesse artigo as etapas para o desenvolvimento e implementação de metodologia para a execução de um estudo padrão de MI. A metodologia apresentada se baseia na identificação petrográfica, uso de platina de ultra-aquecimento, microssonda eletrônica e LAICP-MS. Essas técnicas quando combinadas, permitem a obtenção dos dados composicionais, físico-químicos e genéticos de modo confiável e reprodutível, sendo por isso escolhidas para serem descritas nesse artigo. Dessa forma, espera-se que este seja apenas o início do desenvolvimento de uma série de pesquisas utilizando as MI para a compreensão de processos petrogenéticos e metalogenéticos no Brasil.

Palavras-chave: Melt Inclusions, rochas magmáticas, aplicações, técnicas de estudo.

ABSTRACT: MELT INCLUSIONS: MAIN CHARACTERISTICS AND STUDY TECHNIQUES - Melt inclusions are portions of the melt from which rock was formed, which was entrapped in the crystal irregularities during rock crystallization. Their shapes are varied and reflect their maturity after imprisonment. The distribution of MI depends on the mineral host: can be irregular (in olivine and clinopyroxene), follow the lines of growth (in plagioclase) or be associated with irregularities in the crystal growth (as in quartz). If the MI behaved as a closed system, protected from getting lost or alteration during the ascent of magma to reach the surface, they are a powerful tool for recovery of characteristics of the magma at the time of its imprisonment. For this reason, the Ml have been used in petrological and petrogenetic studies on magmatic systems, or even to understand the processes responsible for mineralization in magmatic-hydrothermal deposits. The study of $\mathrm{Ml}$ allows directly obtaining compositional data and physical-chemical properties of the molten magma at the stage of crystallization of host mineral. Aiming to spread this technique, this paper presents the steps for the development and implementation of a methodology for a standard study of MI. The methodology presented is based on petrographic identification, use of ultra- heating stage, electron microprobe and LA-ICP-MS. These techniques represent the best and most widespread that combined allow to obtain reliably and reproducibly compositional, physical-chemical and genetic data, and are therefore chosen to be described in this article. Thus, it is expected that this is only the beginning of the development of a series of research using the MI for the understanding of petrogenetic and metallogenetic process in Brazil.

Keywords: Melt inclusions, magmatic rocks, applications, study techniques.

\section{INTRODUÇÃO}

Melt inclusions (MI) são pequenas porções de fundido magmático contendo uma combinação de cristais, vidro e vapor que foram aprisionadas nos cristais durante seu crescimento e formação nos magmas. Por esta razão, se elas ficaram preservadas e funcionaram como um sistema isolado, poderão fornecer informações únicas e a possibilidade de reconstituição da composição do magma (fundido silicatado e voláteis) naquele específico estágio de evolução (Frezzotti, 2001).

Este artigo tem como principal objetivo chamar a atenção sobre a importância do estudo das MI, que atualmente é pouco desenvolvido no Brasil. Objetiva-se detalhar as metodologias escolhidas para o desenvolvimento de um estudo padrão de
MI, juntamente com as dificuldades e implicações analíticas inerentes à sua utilização. Também serão descritos os passos seguidos na implementação da metodologia de pesquisa para MI utilizada no Centro de Desenvolvimento da Tecnologia Nuclear (CNEN/CDTN). Este tipo de trabalho também visa estimular outros pesquisadores a se dedicarem ao desenvolvimento dessas técnicas e aplicações nos diferentes ambientes magmáticos, contribuindo para a difusão deste tipo de pesquisa no Brasil.

Na tentativa de explorar as possibilidades que os estudos de MI podem apresentar, na primeira parte deste artigo será feita uma revisão dos conceitos e apresentação das principais técnicas utilizadas. $\mathrm{Na}$ segunda parte, será apresentado o desenvolvimento das técnicas implementadas no Laboratório de Inclusões Fluidas e Metalogênese do CDTN. Além 
disso, serão discutidas as maiores dificuldades encontradas durante a termometria ou na preparação para as análises químicas.

As inclusões magmáticas silicatadas foram descritas e identificadas detalhadamente pela primeira vez por Sorby (1858), e seus desenhos e descrições serviram de base para todos os trabalhos posteriores. Neste trabalho clássico, foram observadas inclusões silicatadas e fluidas em rochas vulcânicas e plutônicas. As inclusões vítreas em lavas foram comparadas às existentes em minerais de escória metalúrgica. Estas inclusões foram descritas como "a mais perfeita analogia entre cavidades preenchidas por vidro em rocha e cavidades preenchidas por fluidos em todos os aspectos, exceto a natureza das substâncias incluídas".

Somente nas últimas décadas, após o desenvolvimento da microssonda eletrônica e outras técnicas analíticas, as MI se tornaram uma importante ferramenta para estudos petrológicos. Nos sistemas magmáticos, o estudo das MI tem como objetivo a caracterização da composição, condições de formação e evolução do magma parental, além da influência dos voláteis (Roedder, 1984; Danyushevsky et al., 2000; Lowenstern, 2003; Sobolev et al., 2009). Entre esses sistemas, a maior parte dos estudos tem sido desenvolvida em rochas vulcânicas, geralmente de idade recente, onde as inclusões são maiores, podendo estar mais bem preservadas.

Já em rochas graníticas, e outras rochas plutônicas, as pesquisas são mais escassas e as dificuldades maiores. O tamanho das inclusões é mais reduzido, e durante o longo tempo de resfriamento das rochas, vários processos de alteração afetam as $\mathrm{MI}$, tais como dissociação dos voláteis e oxidação ou reequilíbrio parcial com o mineral hospedeiro (Danyushevsky et al., 2002). Ainda assim, por meio de uma observação cuidadosa, as MI em sistemas graníticos podem ser usadas para estudos petrogenéticos fornecendo informações sobre os voláteis e as condições de formação (Thomas \& Klemm, 1997). Nos pegmatitos, as pesquisas podem fornecer dados importantes para a compreensão da composição do fundido, da forma de partição dos voláteis e sua influência para o enriquecimento e formação das fases minerais de maior interesse econômico (Roedder, 1984; Thomas et al., 2000; Thomas et al., 2011).

Em depósitos magmáticos hidrotermais, as $\mathrm{MI}$ são amplamente usadas para a compreensão dos processos de exsolução dos voláteis, responsáveis pela formação do minério hidrotermal em depósitos tipo cobre pórfiro (Halter et al, 2004; Student \& Bodnar, 2004) ou para a gênese de skarns (Zhao et al., 2003).
Embora seja muito importante o número de trabalhos publicados na literatura internacional sobre $\mathrm{MI}$ nos últimos 30 anos, os estudos desenvolvidos no Brasil são raros, já que há poucos pesquisadores dedicando-se ao tema. Nesse sentido é bom destacar que a primeira dissertação brasileira sobre $\mathrm{MI}$ foi recentemente defendida na Universidade de Brasília (Silva, 2012). Nesse trabalho é apresentada uma excelente discussão sobre a metodologia de estudos de Laser Ablation acoplado ao Inductively coupled plasma mass spectrometry (LA-ICP-MS) em MI e testes termométricos de alta temperatura desenvolvidos nos EUA.

\section{TÉCNICAS PARA ESTUDO DAS MELT INCLUSIONS}

No estudo de MI podem ser utilizadas diversas técnicas analíticas com o intuito de quantificar, qualificar e caracterizar sua ocorrência. As principais técnicas são:

- Petrografia;

- Termometria de alta-temperatura;

- Microssonda eletrônica;

- Espectroscopia micro-Raman;

- Espectroscopia micro-Infravermelho;

- LA-ICP-MS.

Todas essas técnicas apresentam vantagens e desvantagens sendo apresentado na Tabela 1 um sumário delas.

Serão enfatizadas a petrografia, a termometria de alta temperatura, microssonda eletrônica e LAICP-MS como técnicas principais para o desenvolvimento de um estudo padrão de MI. Estas técnicas são as melhores e mais difundidas. Quando combinadas, permitem obter os dados químicos e microquímicos, físico-químicos e genéticos de modo confiável e reprodutível.

\section{PETROGRAFIA}

Schiano (2003) definiu as MI silicatadas como pequenos volumes (<300 $\mu \mathrm{m}$ de diâmetro) de fundido silicatado que são aprisionados nos minerais durante o seu crescimento. Assume-se que o fundido magmático aprisionado no mineral fica protegido de perdas durante a ascensão. Ele pode reter altas concentrações de voláteis, que podem ser usadas para estimar a pressão de saturação do gás. Nos sistemas vulcânicos, as MI silicatadas consistem de vidro \pm gás \pm cristais. Em rochas plutônicas é mais difícil identificá-las, uma vez que podem estar completamente cristalizadas (Roedder, 1984) ou ter sido alteradas por processos hidrotermais tardios (Varela, 1994; Student \& Bodnar, 2004). 
Qualquer estudo bem sucedido das MI silicatadas deve ser precedido por investigações petrográficas cuidadosas (Frezzotti, 2001). Tamanho, forma, coloração, distribuição no cristal são algumas das características principais que devem ser observadas. Quando o mineral hospedeiro é translúcido e relativamente claro, as $\mathrm{Ml}$ podem ser observadas ao microscópio petrográfico com magnificação média a alta (objetivas maiores que 10X) em luz transmitida.

Tabela 1 - Comparação entre as técnicas analíticas empregadas no estudo de MI (adaptado de Frezzotti, 2001)

\begin{tabular}{|c|c|c|}
\hline Metodologia & Dados obtidos & Dificuldades \\
\hline $\begin{array}{l}\text { Microtermometria } \\
\text { de Alta } \\
\text { temperatura }\end{array}$ & $\begin{array}{l}\text { - Temperatura de } \\
\text { fusão das fases } \\
\text { cristalizadas; } \\
\text { - Temperatura de } \\
\text { homogeneização } \\
\text { total; } \\
\text { - Revitrificação do } \\
\text { conteúdo da Ml; } \\
\text { - Identificação de } \\
\text { processos de } \\
\text { imiscibilidade. }\end{array}$ & $\begin{array}{l}\text { Superestimativa } \\
\text { da temperatura. }\end{array}$ \\
\hline $\begin{array}{l}\text { Microssonda } \\
\text { Eletrônica }\end{array}$ & $\begin{array}{l}\text { - Composição } \\
\text { química do vidro } \\
\text { constituinte da } \mathrm{MI} \text { e } \\
\text { presença de } \mathrm{Cl}, \mathrm{F}, \mathrm{S} \text {, } \\
\text { e P; } \\
\text { - Estimativa da } \\
\text { concentração de } \\
\mathrm{H}_{2} \mathrm{O} \text {. }\end{array}$ & $\begin{array}{l}\text { Necessidade de } \\
\text { homogeneização } \\
\text { prévia quando } \\
\text { as MI possuem } \\
\text { minerais } \\
\text { cristalizados. } \\
\text { As MI } \\
\text { necessitam estar } \\
\text { expostas na } \\
\text { superfície. } \\
\text { Possibilidade de } \\
\text { migração de } \\
\text { parte de seus } \\
\text { constituintes } \\
\text { químicos. }\end{array}$ \\
\hline $\begin{array}{l}\text { Espectroscopia } \\
\text { micro-Raman }\end{array}$ & $\begin{array}{l}\text { - Quantificação dos } \\
\text { gases presentes; } \\
\text { - Caracterização } \\
\text { dos minerais } \\
\text { cristalizados. }\end{array}$ & $\begin{array}{l}\text { Dificuldade para } \\
\text { realização da } \\
\text { quantificação } \\
\text { em sólidos. }\end{array}$ \\
\hline $\begin{array}{l}\text { Espectroscopia } \\
\text { microinfravermelho }\end{array}$ & $\begin{array}{l}\text { - Quantificação da } \\
\text { presença de } \mathrm{H}_{2} \mathrm{O} \text {, } \\
\mathrm{OH}^{-}, \mathrm{CO}_{2} \text { e }\left(\mathrm{CO}_{2}\right)^{2-} \text { no } \\
\text { vidro constituinte } \\
\text { da } \mathrm{MI} \text {. }\end{array}$ & $\begin{array}{l}\text { Padrões para } \\
\text { calibração da } \\
\text { medida e } \\
\text { preparação da } \\
\text { amostra, sendo } \\
\text { necessário o } \\
\text { conhecimento } \\
\text { da espessura da } \\
\text { MI. }\end{array}$ \\
\hline LA-ICP-MS & $\begin{array}{l}\text { - Composição } \\
\text { química da MI; } \\
\text { - Constituição dos } \\
\text { elementos traços da } \\
\text { MI. }\end{array}$ & $\begin{array}{l}\text { Necessidade do } \\
\text { conhecimento } \\
\text { da profundidade } \\
\text { em que se } \\
\text { encontram as } \\
\text { MI, quando não } \\
\text { estão expostas. }\end{array}$ \\
\hline
\end{tabular}

Todos os esforços devem ser feitos para examinar as MI no mineral hospedeiro, embora isto nem sempre seja possível. Índice de refração no óleo ou etanol pode ser usado para aumentar a quantidade de luz passando a partir do mineral. Mesmo usando um aumento adequado e todas as propriedades óticas, nem sempre é possível fazer a distinção entre as $\mathrm{Ml}$ e pequenas inclusões minerais usando somente microscópio ótico, mesmo os de alta definição. Algumas vezes, quando o mineral hospedeiro está coberto por uma fina camada de vidro, matriz ou produtos de alteração, o uso de seções espessas duplamente polidas, é o mais recomendado (Kent, 2008).

Quando a inclusão é cogenética com o mineral hospedeiro é classificada como primária; se foi formada após o mineral, é secundária (Fig. 1). As inclusões primárias são pequenas quantidades do fundido incluídas nos minerais durante 0 crescimento ou recristalização da estrutura do cristal (Schiano, 2003). As MI primárias se formam por numerosas condições relacionadas a irregularidades no crescimento dos cristais, incluindo defeitos no crescimento devido a diferentes processos (como estagnação, undercooling, kinking) e também por imiscibilidade da fase fluida ou aprisionamento de inclusões sólidas (Roedder, 1984; Frezzotti, 2001).
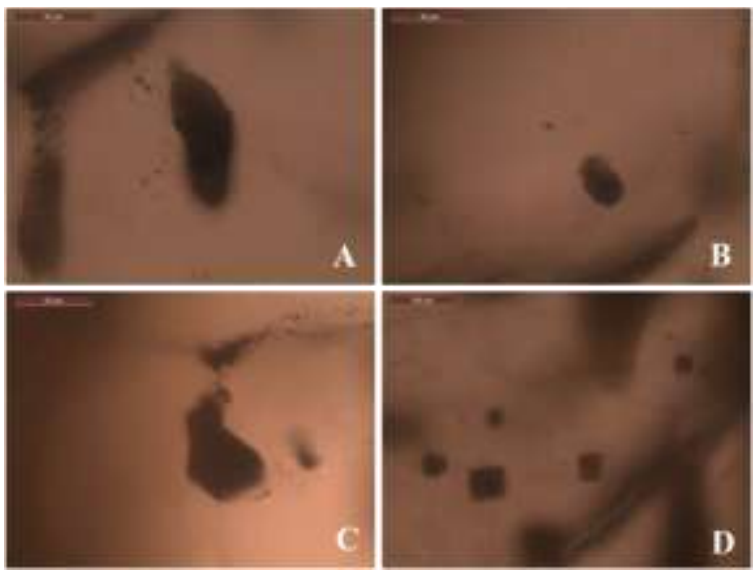

Figura 1 - Diferentes formas das MI primárias: A) alongadas; $B$ ) arredondadas; C) Cristal de quartzo com golfos de corrosão. ou D) cristais negativos. Fenocristais de plagioclásio e quartzo de tufos e andesitos do Grupo Iricoumé, AM.

As inclusões secundárias são localizadas em fraturas que cortam todas as zonas de crescimento dos cristais, as geminações e os planos de clivagem. A feição mais característica das MI secundárias é a ausência de relação entre o fundido aprisionado e os processos de crescimento do cristal hospedeiro. Fraturas lineares que podem cortar vários minerais são indicativas de que os cristais passaram por eventos de deformação cisalhante, foram invadidas pelo fundido magmático e depois seladas, por causa dos processos de aquecimento, tornando-as invisíveis. As inclusões secundárias geralmente possuem formas esféricas ou subesféricas, mas podem ser também elipsoidais, achatadas, alongadas ou ameboides se passaram por algum processo de deformação (Schiano, 2003).

Geralmente, do ponto de vista morfológico, as MI são pseudoarredondadas ou "suavizadas", seguindo a simetria do mineral hospedeiro. As 
inclusões que apresentam forma de cristais negativos foram consideradas resultantes do reequilíbrio com o mineral hospedeiro (Viti \& Frezzotti, 2001). Estas morfologias refletem a sua maturação pós-aprisionamento, em que há difusão entre a superfície da inclusão e o mineral hospedeiro, gerando uma diminuição da área superficial por volume. Entretanto, trabalhos experimentais mostram que a variação na morfologia não modifica a composição das $\mathrm{MI}$, que pode ser considerado representativo do magma aprisionado (Manley, 1996).

A distribuição das $\mathrm{MI}$ dentro dos cristais depende também do mineral hospedeiro. Em cristais de olivina e clinopiroxênio as MI têm uma tendência a uma distribuição irregular. Entretanto, no plagioclásio as $\mathrm{MI}$ se alinham nas zonas de crescimento do cristal. Nos cristais de quartzo, a distribuição pode ser controlada por irregularidades no crescimento, como crescimento esqueletal e corrosão em forma de golfos (Frezzotti, 2001; Schiano, 2003) (Fig. 2).

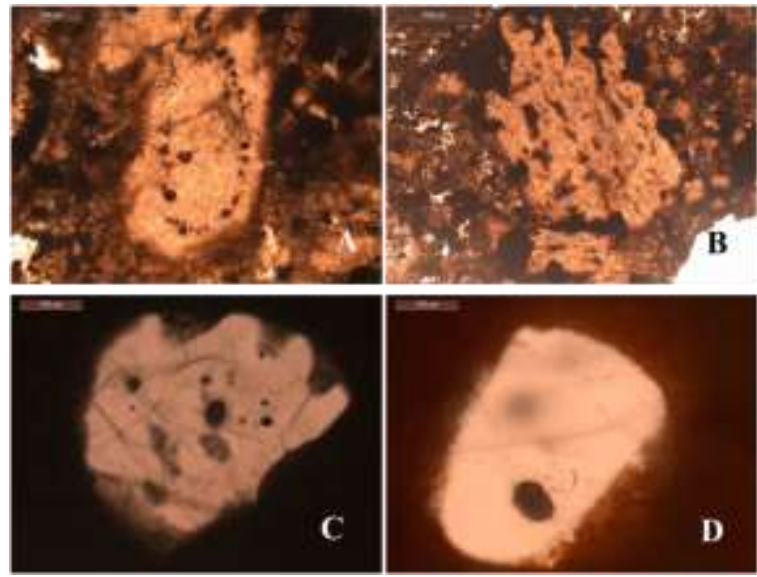

Figura 2 - Distribuição das MI no mineral hospedeiro. A) MI seguem as linhas de crescimento do cristal do plagioclásio. B) MI distribuem-se principalmente nos planos de clivagens e nas bordas de um cristal de plagioclásio. C) Cristal de quartzo com corrosão em golfos e distribuição aleatória das MI. D) Cristal de quartzo arredondado contendo apenas uma MI grande.

Bodnar \& Student (2006) sugeriram que sejam adotados para os estudos das $\mathrm{Ml}$ os mesmos princípios usados para as inclusões fluidas. Foi introduzido o conceito de melt inclusions assemblage (MIA) para identificar os grupos de $\mathrm{MI}$ que foram aprisionados ao mesmo tempo, e por analogia, na mesma temperatura e pressão e com fundido magmático com a mesma composição. Assim, as MI que ocorrem ao longo das zonas de crescimento de um cristal individual podem representar MIA contendo MI primárias. Se comparadas com as inclusões fluidas, as $\mathrm{MI}$ secundárias são muito menos comuns. No mínimo, as $\mathrm{MI}$ num dado fenocristal podem ser consideradas como uma MIA composta de inclusões que foram todas aprisionadas durante um período de tempo (desconhecido), necessário para o crescimento do cristal. Quando as MI ocorrem ao longo das superfícies de crescimento, ou outras interfaces do cristal, fica fácil associá-las como pertencentes à mesma MIA (Bodnar \& Student, 2006).

Entretanto, em muitos minerais ou fenocristais existem apenas poucas $\mathrm{MI}$, que aparecem distribuídas irregularmente. Neste caso, as observações petrográficas não são determinantes para dizer se elas pertencem à mesma MIA. Se elas tiverem características petrográficas similares, elas podem pertencer ao mesmo grupo.

\section{MODIFICAÇÕES APÓS O APRISIONAMENTO:}

As MI podem variar após a formação quanto à forma, tamanho, formação da bolha, composição, cristalinidade e fraturamento. Embora a maior parte destas variações tenha sido observada nas MI estudadas, nem todas anulam sua aplicabilidade. As variações na forma, inclusive com a formação de cristais negativos, são interpretadas como resultante do processo de anneling, que diminui a energia superficial. Entretanto, nesse caso, a variação na forma não altera a composição da $\mathrm{Ml}$ ((Danyushevsky et al., 2000, Frezzotti, 2001).

Pode ocorrer, ainda, uma cristalização incipiente, ou reabsorção do mineral hospedeiro, na parede da inclusão. Danyushevsky et al. (2002) explicam que isto ocorre porque a parede da inclusão funciona como fase de borda com baixa energia de nucleação. A composição da $M I$ pode ser ajustada em relação à composição mineral hospedeiro (Danyushevsky et al., 2000; Webster \& Duffield, 1991). Trabalhos experimentais desenvolvidos em MI no plagioclásio mostram que a concentração de água na camada de borda é muito similar à encontrada na composição total do fundido magmático, por causa do seu alto coeficiente de difusão (Baker, 2008). Entretanto, para os elementos $\mathrm{S}$ e $\mathrm{P}$ com baixo coeficiente de difusão, a concentração na MI pode ser superestimada em relação aos seus valores no fundido original. Assim, nas $\mathrm{Ml}$, outros elementos com coeficientes de difusão similares ao enxofre podem mostrar enriquecimento em relação à composição total do fundido magmático (Baker, 2008).

Com a diminuição das temperaturas magmáticas, os processos que mais afetam as MI são cristalização e desvitrificação. A cristalização ocorre quando se formam fases cristalinas a partir do fundido, resultando da reorganização parcial do fundido para formar micrólitos. Nesse caso, a inclusão aparece escura ou opaca, com aspecto granular, e a observação dos cristais não pode ser feita por microscopia ótica em luz transmitida (Fig. 3). A 
desvitrificação torna o vidro colorido, mostrando uma anisotropia pontual.
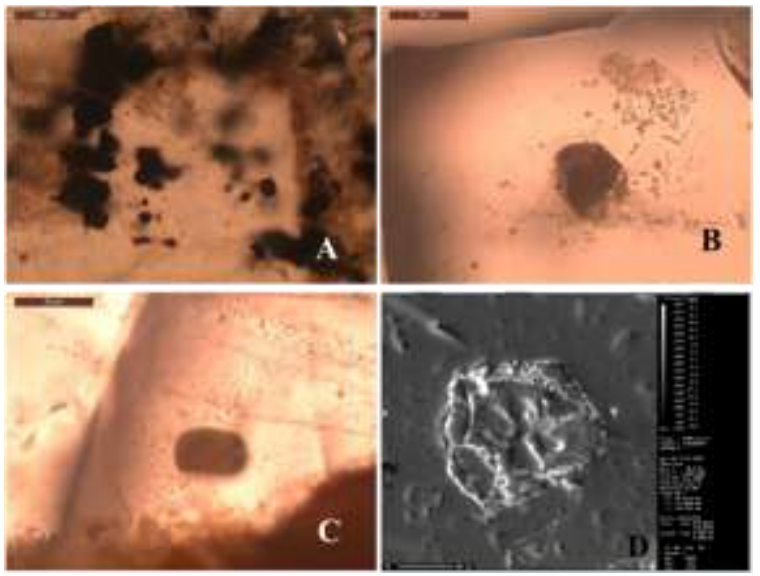

Figura 3 - As principais características das MI afetadas por recristalização são a aparência escura ou opaca (A) e com aspecto granular (B), quando observadas ao microscópio ótico. $A$ coloração, geralmente amarronzada, pode estar relacionada ao processo de desvitrificação (C). Uma MI recristalizada, quando observada ao microscópio eletrônico pode ser formada por diversos microcristais diferentes $(D)$, como quartzo, apatita, titanita e clorita.

O grau de evolução da MI depende do tamanho da inclusão e do tempo de resfriamento. Em lavas com resfriamento rápido, as $\mathrm{MI}$ não desvitrificam ou cristalizam. A razão de resfriamento após o aprisionamento é muito mais importante que a idade absoluta da rocha (Frezzotti, 2001). Processos de cristalização e desvitrificação são observados com mais frequência em $\mathrm{Ml}$ de rochas intrusivas, tornando seu estudo muito mais complexo.

\section{TERMOMETRIA}

A termometria tem como objetivos:

a. homogeneizar a $\mathrm{MI}$, revertendo o processo de cristalização a partir da refusão do seu conteúdo e

b. fornecer informações termométricas sobre a temperatura mínima de cristalização do mineral hospedeira (Sobolev \& Shimizu, 1993).

Dessa forma, a premissa mais importante a ser seguida é que a Ml seja um sistema isocórico. Portanto as inclusões não podem estar expostas na superfície ou conectadas a fraturas, clivagens e outros aspectos que indiquem que o sistema MI não se encontra mais isolado.

A necessidade das $\mathrm{MI}$ serem isocóricas também é dependente de sua homogeneização, pois durante o resfriamento parte do fundido magmático que compõe a $\mathrm{Ml}$ pode cristalizar nas paredes da inclusão o mesmo tipo de mineral do hospedeiro, dessa forma mudando as condições internas (composição e pressão). Caso essa situação seja observada, prossegue-se com a termometria, mas havendo a necessidade de observar que esse material cristalizado nas paredes deve-se fundir, para que o sistema volte a ter as condições originais e o resultado obtido corresponda a essa situação. De forma semelhante, a afirmação anterior não será atendida caso as bordas das inclusões sejam deformadas ou absorvidas pelo fundido (Frezzotti, 2011). Relacionando as transformações ocorridas dentro da $\mathrm{Ml}$ com temperatura/pressão interna na MI é possível traçar o diagrama da figura 4.

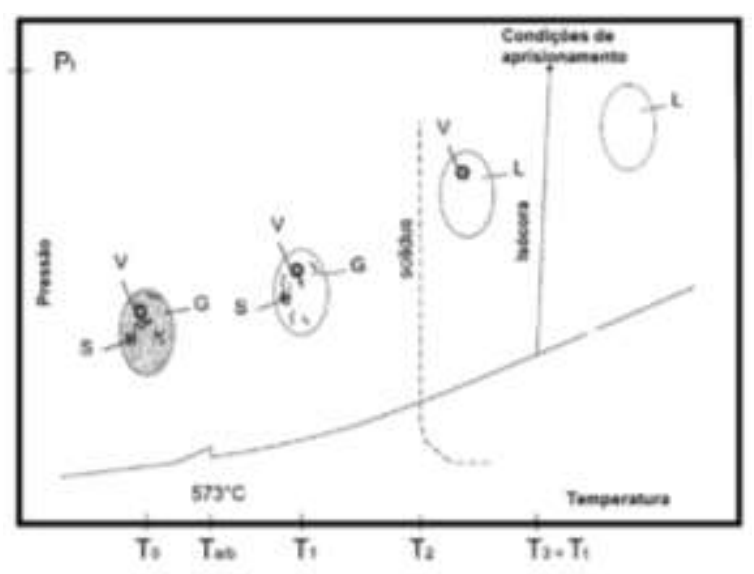

Figura 4 - Diagrama de transformações ocorridas em relação à temperatura/pressão (adaptado de Frezzotti, 2001).

Várias técnicas diferentes têm sido usadas para homogeneizar as $\mathrm{Ml}$, podendo ser divididas naquelas em que as MI são aquecidas a uma atmosfera de pressão confinante, ou a pressão elevada. Podem ainda ser divididas nas técnicas em que o aquecimento é feito continuamente em um passo ou em vários incrementos (Bodnar \& Student, 2006).

A técnica mais comumente usada é a homogeneização por meio de platinas termométricas acopladas a microscópios petrográficos onde é possível observar as mudanças de fase durante o aquecimento (Sobolev et al., 1980, Magakyan et al., 1993; Lowenstern et al., 1994; Reyf, 1997; Zhao et al., 2003, Schiano, 2003). A temperatura de homogeneização do conteúdo da inclusão (gás, vidro e cristais) para uma fase fundida uniforme, Th, é a temperatura mínima de aprisionamento do fundido magmático no cristal hospedeiro. A correção da pressão pode ser feita para se obter a temperatura real de aprisionamento, como nas inclusões fluidas (Roedder, 1984). A temperatura de início de fusão, Ti, reflete a temperatura do solidus.

Podem ocorrer diferenças importantes entre as condições físico-químicas do fenocristal e qualquer hospedeiro da $\mathrm{Ml}$ entre a cristalização e as condições experimentais. Por exemplo, a pressão no fenocristal durante o aquecimento a $1 \mathrm{~atm}$ é sempre menor que durante o resfriamento natural. A 
pressão dentro da $\mathrm{Ml}$ tende a aumentar com o aumento da temperatura. Assim, a razão de aquecimento para $\mathrm{Ml}$ dos diferentes tipos de magmas, pode ser diferente para os diferentes tipos de fenocristais (Danyushevsky et al., 2002).

Outro método de aquecimento utiliza uma autoclave, em que as amostras contendo as MI são aquecidas a temperaturas superiores a esperada para o solidus. Posteriormente, são resfriadas e examinadas ao microscópio para verficar evidências de homogeneização (Thomas \& Klein, 1997, Peretyazhko et al., 2004). Os pesquisadores que utilizam esta técnica defendem que ela é mais precisa, porque a homogeneização da $\mathrm{Ml}$ é lenta. $\mathrm{O}$ que geralmente é medido é a temperatura de homogeneização aparente, que depende da razão e duração do aquecimento e do tamanho da inclusão.

A microtermometria convencional leva a obtenção de uma temperatura de homogeneização superestimada. Com uso da autoclave pode-se utilizar um novo parâmetro, chamado diâmetro crítico $\left(\mathrm{d}_{\mathrm{K}}\right)$. Após o resfriamento, as $\mathrm{Ml}$ menores podem estar completamente homogeneizadas, enquanto as grandes não, e algumas de tamanho intermediário estarão no ponto adequado de homogeneização. Estas últimas são as inclusões críticas, e seus tamanhos são cuidadosamente medidos. Dependendo da abundância das inclusões é fácil medir um grande número delas em cada amostra após o experimento. Isto é importante para reduzir os efeitos causados por perda de água por difusão durante o aquecimento, assim como as diferenças nas histórias de resfriamento e cristalização (Thomas, 1992; Thomas et al., 1996; Thomas \& Klemm, 1997).

Para MI grandes (>100 $\mu \mathrm{m})$, pode-se utilizar uma autoclave com pressão externa de água, em que seu volume é calculado para atingir 2 a 2,5 kbar na temperatura final. Para evitar dissolução significativa do conteúdo da $\mathrm{Ml}$, usa-se mineral hospedeiro moído junto com os fragmentos na ampola (Peretyazhko et al., 2004).

Como todas as técnicas, ambas apresentam vantagens e desvantagens. A comparação entre elas pode ser visualizada na tabela 2 .

Tabela 2 - Comparação entre dois meios de se realizar a termometria das MI (Adaptado de Frezzoti, 2001).

\begin{tabular}{lcc}
\hline & $\begin{array}{c}\text { Platina } \\
\text { termometria }\end{array}$ & Forno \\
\hline Preparação de amostra & Sim & Não \\
Grande quantidade de amostra & Não & Sim \\
Observação das transições de fases & Sim & Não \\
Controle de temperatura direta & Sim & Não \\
\hline
\end{tabular}

Independentemente da metodologia utilizada durante a termometria, podem surgir alguns problemas. Por exemplo, quando se realiza o aquecimento das amostras pode ocorrer a difusão dos voláteis. Dessa forma, durante os estudos termométricos, é necessário ter em mente que os voláteis e outros elementos, que podem se difundir para o mineral hospedeiro, serão subestimados (Lowenstern, 1995).

\section{MICROSSONDA ELETRÔNICA}

A microssonda eletrônica é a principal técnica não destrutiva utilizada para quantificação dos constituintes das MI. Quando as MI não são vítreas e homogêneas é necessário realizar a homogeneização do conteúdo por meio de refusão por microtermometria. Posteriormente, as MI devem ser expostas na superfície do mineral, a partir do desbaste da amostra.

$\mathrm{Na}$ microssonda é possível quantificar os elementos maiores e traço (acima de 500 ppm), inclusive $\mathrm{F}, \mathrm{Cl}$, S e P. A quantificação da $\mathrm{H}_{2} \mathrm{O}$ presente nas $\mathrm{Ml}$ pode ser realizada apenas por inferência, o que dá uma ideia se o fundido original era anidro ou não (Anderson, 1974). Outro limitante nessa técnica é a impossibilidade de quantificar o $\mathrm{CO}_{2}$.

Pode ainda haver a migração de componentes, a exemplo do $\mathrm{Na}$, das $\mathrm{Ml}$ para o hospedeiro, o que influencia a estimativa da quantidade verdadeira de água presente no fundido original (Nielsen \& Sigurdsson, 1981). Este problema da migração pode ser contornado com ajustes na rotina de análise, como diminuição da corrente de incidência, alargamento do feixe e ordenamento dos elementos para análises (Nielsen \& Sigurdsson, 1981).

\section{LA-ICP-MS}

A utilização de LA-ICP-MS está na vanguarda nos estudos de MI. Esta técnica permite a obtenção de resultados rápidos, sem a necessidade de preparações complicadas. A partir dessa metodologia é possível dosar não somente os elementos traço, como também os que estão em quantidades maiores nas MI (Halter et al., 2002; Pettke et al. , 2004). Taylor et al. (1997) afirmam que tal técnica possui duas características ótimas para analises de MI: grande resolução espacial e grande sensibilidade.

O uso desse equipamento permite a deteç̧ão e determinação em ampla faixa de concentração, de $\% \mathrm{~m} / \mathrm{m}$ à $\mathrm{ng} / \mathrm{g}$, juntamente com precisão de $2-5 \%$ para todos os elementos existentes (Heinrich et al., 2003).

O LA-ICP-MS dispensa a necessidade de homogeneizar previamente as MI. Entretanto, é necessário que estejam bem próximas da superfície. As únicas informações realmente importantes para a 
realização do estudo é o conhecimento da profundidade da $\mathrm{Ml}$, seu tamanho e a composição do mineral hospedeiro.

Os elementos com maior potencial de ionização, $\mathrm{H}, \mathrm{N}, \mathrm{O}, \mathrm{Cl}$ e gases nobres, possuem grandes problemas para serem quantificados devido a interferências em relação ao background e formação de íons diatômicos (Pettke, 2006). A desvantagem é o fato de ser uma técnica destrutiva de análise, impedindo a repetição da análise na mesma região ou inclusão. A avaliação estatística da confiabilidade da análise é contornada quando há MIA, onde admite-se que as MI tenham composição similares.

\section{MELT INCLUSIONS NO LABORATÓRIO DE INCLUSÕES FLUIDAS E METALOGÊNESE-CDTN}

\section{Equipamentos Utilizados}

No Laboratório de Inclusões Fluidas e Metalogênese (LIFM) do CNEN/CDTN, a rotina de estudo de Ml está sendo desenvolvida e aprimorada desde 2007 (Lima et al., 2007). Os estudos iniciaramse com a calibração e colocação em rotina de uma platina de alta temperatura Leitz Heating Stage 1350 (Fig 5). Paralelamente, o aquecimento também tem sido feito em autoclaves e os resultados do aquecimento comparados. A platina acoplada ao microscópio ótico LEITZ Ortholux IIPOL, pode ser refrigerada por água ou gás $(\mathrm{N}, A r)$. Outra característica relevante está ligada ao controle da taxa de aquecimento, que pode variar de 1 a $10 \stackrel{\circ}{C}$ por minuto (limite imposto pelo fabricante), realizada a partir do incremento na corrente elétrica.

Para o desenvolvimento da metodologia de identificação química das $\mathrm{MI}$ foi utilizada uma microssonda eletrônica JEOL modelo JXA-8900RL do consórcio UFMG-CDTN.

O estudo microquímico composicional das $\mathrm{MI}$, minerais e outros materiais poderá ser realizado num futuro próximo. Recentemente, o LIFMCDTN/CNEN adquiriu sistema de Laser Ablation New Wave 213, que já está acoplado num ICP-MS, modelo ELAN DRC-e 6100. Esse sistema encontra-se, atualmente, na fase de otimização e definição das rotinas analíticas para suas diversas aplicabilidades. $\mathrm{O}$ estudo microcomposicional das $\mathrm{Ml}$, minerais e outros materiais poderá ser realizado com confiabilidade num futuro próximo.
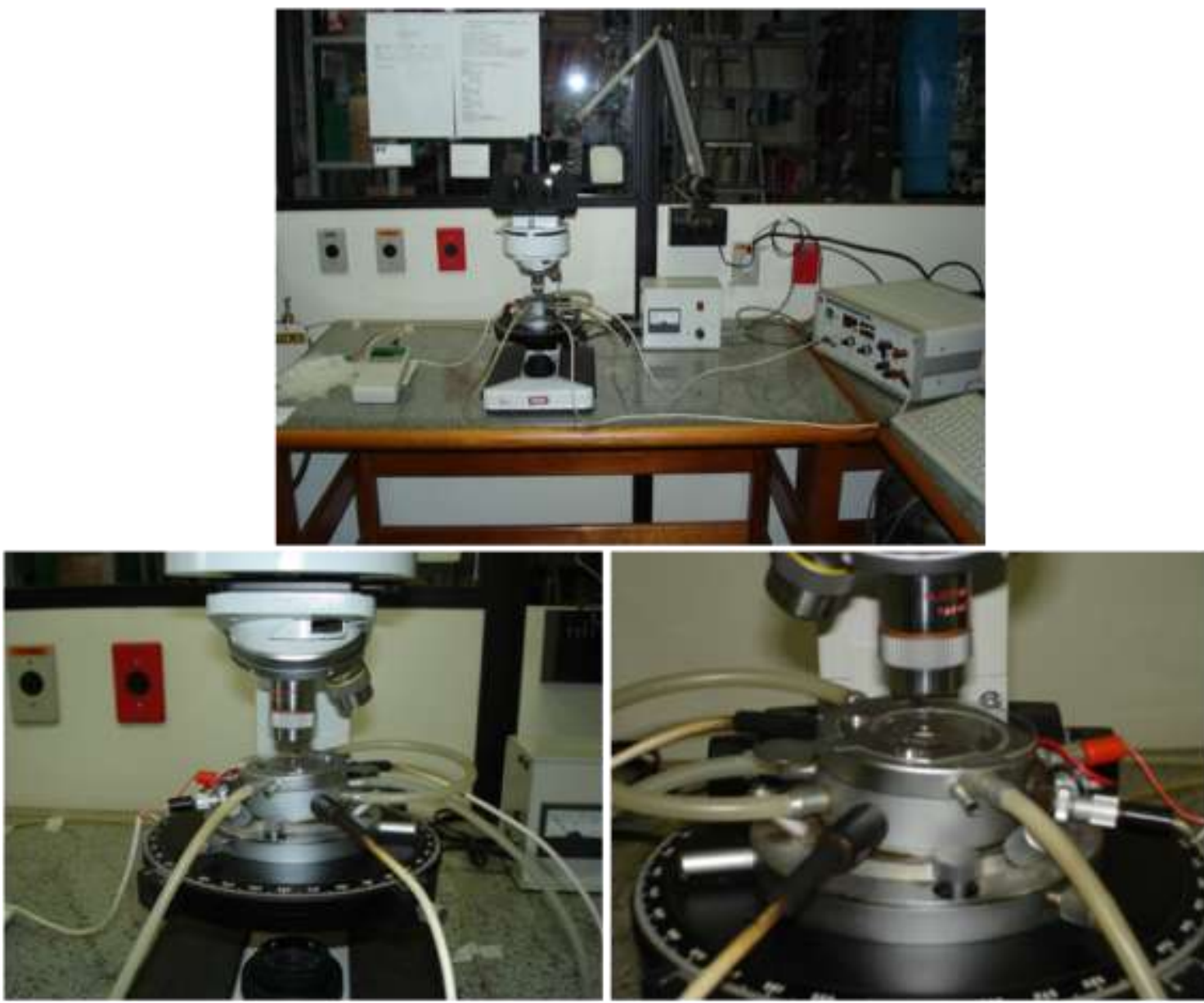

Figura 5 - Platina de aquecimento Leitz Heating Stage 1350 acoplada ao microscópio LEITZ LABORLUX 12 POL. 


\section{RESULTADOS}

Para se atingir uma maior confiabilidade, precisão e reprodutibilidade, nas medidas obtidas a partir da platina HS 1350, foi realizada a calibração do aparelho a partir de ensaios em triplicata, com padrões analíticos que apresentam pontos de fusão distintos no intervalo de temperatura entre 30 e $1300{ }^{\circ} \mathrm{C}$ (faixa de interesse) (Tab. 3). A curva de calibração construída a partir dos ensaios (Fig. 6) exibe linearidade de $R^{2}=0,998$. Esse resultado foi considerado satisfatório, levando-se em consideração a amplitude da faixa de linearidade e indica que a temperatura de fusão teórica e prática estão próximas, com erros inferiores a $5 \%$.

Tabela 3 - Padrões analíticos com seus respectivos pontos de fusão teóricos, a média dos resultados obtidos experimentalmente em triplicata na platina de aquecimento Leitz Heating Stage 1350 e erros percentuais respectivos.

\begin{tabular}{cccc}
\hline $\begin{array}{c}\text { Padrões } \\
\text { analíticos }\end{array}$ & $\begin{array}{c}\mathbf{T}_{\mathbf{f}} \text { teórica } \\
\left({ }^{\circ} \mathbf{C}\right)\end{array}$ & $\begin{array}{c}\mathbf{T}_{\mathbf{f}} \text { prática } \\
\text { média }\left({ }^{\circ} \mathbf{C}\right)^{\mathbf{a}}\end{array}$ & $\begin{array}{c}\text { Erro percentual } \\
(\%)\end{array}$ \\
Padrão 1 & 135,0 & 132,4 & 1,9 \\
Padrão 2 & 306,9 & 313,6 & 2,2 \\
$\mathrm{~K}_{2} \mathrm{Cr}_{2} \mathrm{O}_{7}$ & 394,0 & 406,3 & 3,1 \\
$\mathrm{Ba}\left(\mathrm{NO}_{3}\right)_{2}$ & 592,0 & 584,7 & 1,2 \\
$\mathrm{LiF}$ & 870,0 & 871,4 & 0,2 \\
$\mathrm{Cu}$ & 1084,6 & 1138,1 & 4,9 \\
\hline \multicolumn{4}{c}{ a. média da faixa de temperatura de fusão }
\end{tabular}

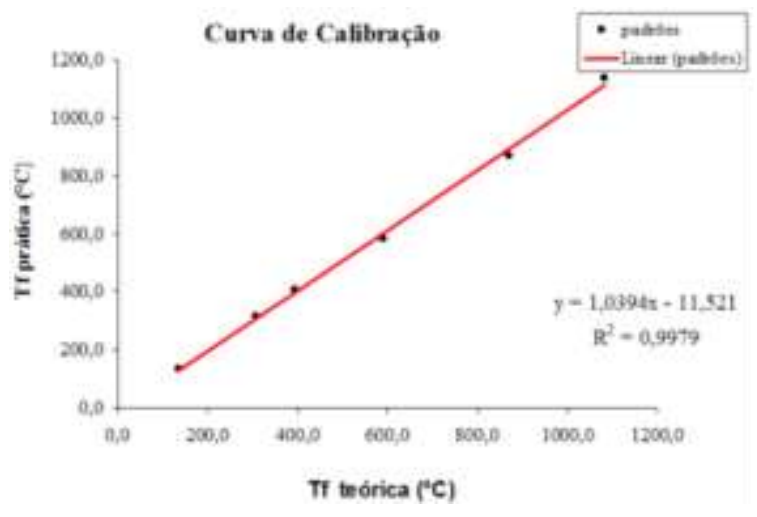

Figura 6 - Gráfico da curva de calibração da platina de aquecimento Leitz Heating Stage 1350 obtido a partir dos padrões analíticos.

Para desenvolver 0 conjunto de técnicas utilizadas no estudo rotineiro de $\mathrm{Ml}$ foram selecionadas amostras de ignimbrito da Formação Chon Aike, Macizo del Deseado, Patagônia, Argentina. As $\mathrm{Ml}$ estavam muito bem preservadas, hospedadas em cristais de quartzo, sem zonas definidas, arredondadas, incolores e bifásicas (vidro - vapor). A bolha corresponde em média a $15 \%$ do volume da inclusão e o seu diâmetro, geralmente, apresenta $80 \mu \mathrm{m}$ (Fig 7).

Para análise na microssonda, as $\mathrm{Ml}$ selecionadas foram expostas na superfície, a partir do desbaste do cristal. Os resultados obtidos com essa metodologia indicaram que a Ml é rica em sílica e que o magma possuía composição riolítica, peraluminosa, com altos teores de $\mathrm{K}$.

Os estudos microtermométricos nas $\mathrm{MI}$ selecionadas tiveram como objetivo identificar e visualizar as transformações de fase, que poderiam servir de base para estudos em amostras com MI menores ou mais complexas. Portanto, foram desenvolvidos para observar o início de fusão, a homogeneização, e determinar as melhores condições de aquecimento na execução das análises.

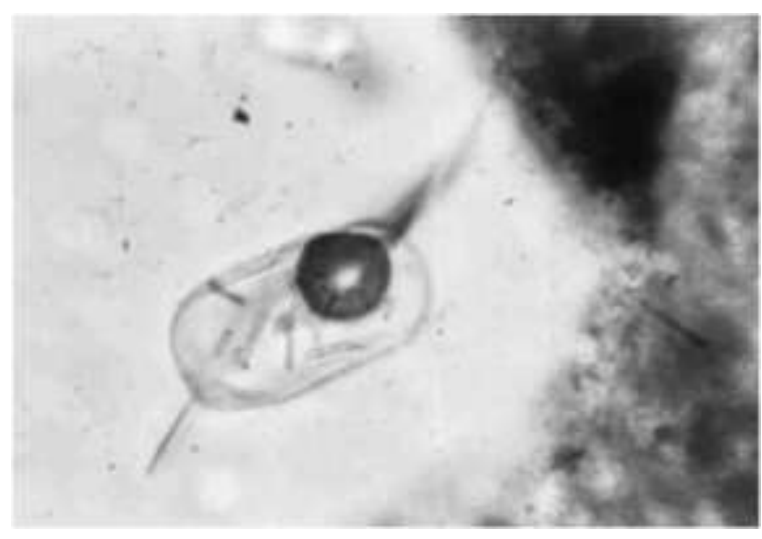

Figura 7 - Ml inclusa em quartzo de Ignimbrito da Formação Chon Aike, Macizo del Deseado, Patagônia,Argentina. A fase clara da $\mathrm{MI}$ é composta por SI, Al, Ke Na, enquanto a faze opaca por $\mathrm{Si}, \mathrm{Al}$, $K, \mathrm{Na}, \mathrm{Ca}, \mathrm{Fe}$ e $\mathrm{S}$.

Outras amostras utilizadas para colocação em rotina das técnicas associadas à $\mathrm{Ml}$, correspondem a cristais de quartzo do granito São Timóteo (Lagoa Real-BA) e fenocristais de plagioclásio e quartzo de rochas vulcânicas do Supergrupo Uatumã. (AM).

A partir destes trabalhos experimentais foram verificadas também várias situações onde pode ocorrer a perda da MI. Cristais de quartzo hospedeiros podem fraturar durante o aquecimento, e se esta fratura atingir a MI haverá perda do seu conteúdo e integridade durante a refusão. Cristais de plagioclásio suportam bem temperaturas elevadas (acima de 1100 으) de refusão de MI. Porém, se o cristal estiver parcialmente alterado ele pode não resistir ao resfriamento rápido, pulverizando-se.

Outra dificuldade verificada é a preservação da MI durante o desbaste e sua exposição na superfície do cristal para análise via microssonda eletrônica. Mesmo com desbaste cuidadoso, usando abrasivo extrafino $[0,25(1 / 4) \mu \mathrm{m}]$ pode haver dificuldades para se obter uma superfície plana e com polimento adequado para as análises, seja por abertura das fraturas, desagregação do cristal, ou devido à diferença de dureza entre o hospedeiro e a MI.

Os resultados obtidos nas amostras dos ignimbritos parecem ser bastante confiáveis. Os testes foram reproduzidos em diferentes $\mathrm{Ml}$ da 
mesma amostra, fornecendo resultados semelhantes. Atualmente estamos desenvolvendo novos testes em rochas graníticas e vulcânicas brasileiras, bem preservadas, ou submetidas a incipientes graus de deformação, visando determinar o grau de recristalização das $\mathrm{MI}$ e a viabilidade do estudo em rochas muito antigas (Paleoproterozoicas).

\section{CONSIDERAÇÔES FINAIS}

O estudo de $\mathrm{MI}$ é uma ferramenta excelente para obtenção de dados composicionais e físico-químicos do fundido magmático de forma direta. A metodologia de estudo de Ml está bem difundida e consolidada no meio cientifico internacional. Porém, até o momento, essa ferramenta vem sendo pouco empregada na comunidade geológica brasileira.

Neste artigo foram apresentadas as principais técnicas analíticas que podem ser utilizadas para desenvolver pesquisas sobre MI. Esses estudos envolvem uma serie de metodologias bem difundidas e consolidadas no meio cientifico internacional, que permitem a obtenção de dados petroquímicos, composicionais e físico-químicos dos fundidos magmáticos de forma direta. Entretanto, pesquisas desse tipo ainda são raras no Brasil. Espera-se que, em curto prazo, e com a disponibilidade de equipamentos em rotina, o estudo das características de formação das $\mathrm{MI}$, e suas implicações na compreensão genética das rochas (e depósitos minerais associados), se torne uma ferramenta mais difundida dentro da comunidade científica brasileira.

\section{AGRADECIMENTOS}

Agradecemos aos órgãos de fomento, CNPq (Projeto 307.546-11), FAPEMIG (Projeto CAG PPM 00666-11), FINEp REDETEC (Projeto 2715/09) e CAPES pelo apoio financeiro fornecido pela por meio de bolsas aos autores e aquisição de equipamentos. Ao CDTN/CNEN pelo suporte financeiro e a disponibilização da infraestrutura. Agradecemos aos revisores Lena V. Monteiro e Antônio L. Gandini pela leitura crítica e sugestões, que melhoraram a qualidade do texto.

Dedicamos esse artigo ao Dr. José Marques Correa Neves, que integrou ativamente a equipe do LIFM/CDTN desde 2002, incentivando permanentemente nossas atividades, particularmente o desenvolvimento da linha de "melt e fluidos", com sua característica energia, sabedoria e amizade. Obrigado Professor!

\section{REFERÊNCIAS}

Anderson, A.T. 1974. Evidence for a picritic, volatile-rich magma beneath Mt. Shasta, California. Journal of Petrology. 15: 243267.

Baker, D.R. 2008. The fidelity of melt inclusions as records of melt composition. Contributions to. Mineralogy and Petrology., 156: 377-395.

Bodnar, R.J. \& Student, J.J. 2006. Melt Inclusions in plutonic Rocks: Petrography and Microthermometry. Mineralogical Association of Canada, Short Course Series. 36: 1-25.

Danyushevsky, L.V.; McNeill, A.W.; Sobolev, A. 2002. Experimental and petrological studies of melt inclusions in phenocrysts from mantle-derived magmas: an overview of techniques, advantages and complications. Chemical. Geology, 183: 5-24.

Danyushevsky, L.V., Della-Pasqua, F.N., Sokolov, S. 2000. Reequilibration of melt inclusions trapped by magnesina olivine phenocriysts from subduction-related magmas: petrological implications. Contributions to. Mineralogy and. Petrology, 138: 66-83.

Frezzotti, M.L. 2001. Silicate-melt inclusions in magmatic rocks: applications to petrology. Lithos, 55: 273-299.

Halter, W. E., Petke, T., Heinrich, A.C., Rothen-Rutishausen, B. 2002. Major to trace element analysis of melt inclusions by laser-ablation ICP-MS: methods of quantification, Chemical Geology, 183: 63-86.

Halter, W.E.; Pettke, T.; Heinrich, C.A. 2004. Laser-ablation ICP-MS analysis of silicate and sulfide melt inclusions in an andesitic complex I: analytical approach and data evaluation. Contributions to. Mineralogy and. Petrology, 147: 385-396.

Heinrich, C.A., Pettke, T., Halter, W.E., Aigner-Torres, M., Aude'Tat, A., Günther, D., Hattendorf, B., Bleiner, D., Guillong, M., Horn, I., 2003. Quantitative multi-element analysis of minerals, fluid and melt inclusions by laserablation inductively-coupledplasma mass-spectrometry. Geochimica et Cosmochimica Acta 67(18), 3473-3497.

Kent, A.J.R. 2008. Melt inclusions in basaltic and associated volcanic rocks. Reviews in Mineralogy and Geochemistry, 69, 173-231.

Lima, T.A.F., Oliveira, E.K.,, Rios, F.J. 2007. Repositório de Rejeito de Alto Nível (RANR) No Brasil: O Emprego da Platina Leitz HS 1350 no Auxílio para Caracterização Geológica de Áreas Candidatas. In: International Nuclear Atlantic Conference, 2007, Santos.

Lowenstern, J.B. 1994. Chlorine, fluid immiscibility, and degassing in peralkaline magmas from Pantelleria, Italy. American Mineralogist, 79: 353-369.

Lowenstern, J.B. 1995. Applications of silicate melt inclusions to the study of magmatic volatiles. In: J.F.H. Thompson (ed). Magmas, Fluids and Ore Deposits. Mineralogical Association of Canada, Short Course, 23: 71-99.

Lowerstern, J.B. 2003. Melt inclusions come of age: volatiles, volcanoes, and Sorby's legacy. In: B. de Vivo \& R.J. Bodnar (eds): Developments in Volcanology 5, Melt Inclusions in Volcanic Systems: Methods, Aplications and Problems. Elsevier, Amsterdam, 1-21.

Magakyan, R.; Sobolev, A.V.; Zakariadze, G.S.; Kononkova, N.N. 1993. Petrology of evolved boninite magmas: evidence from the Mesozoic lesser Caucasus island arc. Petrology, 1: 378394.

Manley, C.R. 1996. Morphology and maturation of melt inclusions in quartz phenocrysts from Badlands rhyolite lava flow, southwestern Idaho. American Mineralogist. 81: 158-168. 
Nielsen, H.C. \& Sigurdsonn, H. 1981. Quantitative methods of electron microprobe analyses of sodium in natural and synthetic glasses. American Mineralogist. 66: 547-552.

Peretyazhko, I.S., Zagorsky, V.Y., Smirnov, S.Z., Mikhailov, M.Y. 2004. Conditions of pocket formation in the Oktyabrskaya tourmaline-rich gem pegmatite (the Malkhan field, Central Transbaikalia, Russia). Chemical. Geology., 210: 91-111.

Pettke, T., Halter, W.E., Webster, J.D., Aigner-Torres, M.; Heinrich, C.A. 2004. Accurate quantification of melt inclusion chemistry by LA-ICPMS: A comparison with EMP and SIMS and advantages and possible limitations of these methods. Lithos 78, 333-361.

Pettke T. 2006. In situ laser-ablation ICPMS analysis of melt inclusions and prospects for constraining subduction zone magmatism. In: Melt Inclusions in Plutonic Rocks (Webster J. D., ed). Mineralogical Association of Canada. Short Course Series 36: 51-80.

Reyf, F.G. 1997. Immiscible phases of magmatic fluid and their relation to Be and Mo mineralization at the Yermakovka $\mathrm{F}-\mathrm{Be}$ deposit, Transbaikalia, Russia. Chemical. Geology., 210: 4971.

Roedder, E. 1984. Fluid Inclusions. Reviews in Mineralogy, 12. $644 \mathrm{pp}$.

Schiano, P. 2003. Primitive mantle magmas recorded as silicate melt inclusions in igneous minerals. Earth-Science Reviews. 63:121-144.

Silva, C.F.C. 2012. Metodologia de Análise de inclusões Fluidas por LA-ICP-MS e Estudo de Inclusões de líquidos Silicáticos Aplicados ao Depósito de Sn e In do Maciço Granítico Mangabeira - Goiás, GO. Dissertação de Mestrado, Instituto de Geociências, Universidade de Brasília, 187 p.

Sorby, H.C. 1858. On the microscopic structure of crystals, indicating the origin of minerals and rocks. Geology Society of. London Quarterly Journal 14: 453-506.

Sobolev, A.V., Dmitriev, L.V., Barsukov, V.L., Nevsorov, V.N., Slutsky, A.V., 1980. The formation conditions of highmagnesium olivines from the monomineral fraction of luna24 regolith. Proc. 11th Lunar and Planetary Science Conference.1:105-116.

Sobolev, A.V. \& Shimizu, N. 1993. Ultra-depleted primary melt included in olivine from Mid-Atlantic ridge. Nature, 363: 151153.
Sobolev, A.V., Keivolutskaya, N.A., Kuzmin, A.V. 2009. Petrology of the Parental Melts and Mantle Sources of Siberian Trap Magmatism. Petrology, 17: 253-296.

Student, J. J., Bodnar, R. J. 2004. Silicate melt inclusions in porphyry copper deposits: Identification and homogenization behavior, Canadian Mineralogist, 42: 1583-1599.

Taylor, R.P., Jackson, S.E., Longerich, H.P., Webster, J.D., 1997. In situ trace-element analysis of individual silicate melt inclusions by laser ablation microprobe inductively coupled plasma-mass spectrometry (LAM-ICP-MS). Geochimica et Cosmochimica Acta 61(13), 2559-2567.

Thomas, R. 1992. Schmelzeinschlüsse in Mineralen granitoider Gesteine - Abschätzung der Solidus-, Liquidus- und Homogenisierungstemperaturen und drucke. Mitteilungen der Österreichischen Mineralogischen Gesellschaft, 137, 1124.

Thomas, R., Rhede, D., Trumbull, R. B. 1996. Microthermometry of volatile-rich silicate melt inclusions in granitic rocks. Zeitschrift für geologische Wissenschaften.24 (3-4),505-526.

Thomas, R. \& Klemm, W. 1997. Microthermometric Study of Silicate Melt Inclusions in Variscan Granites from SE Germany: Volatile Contents and Entrapment Conditions. Journal of Petrology, 38: 1758-1765.

Thomas, R.; Webster, J.D.; Heinrich, W. 2000. Melt Inclusions in pegmatite quartz: Complete miscibility between silicate melts and hydrous fluids at low pressure. Contributions to. Mineralogy and. Petrology, 139: 394-401.

Thomas, R., Webster, J. D., Davidson, P. 2011. Be-daughter minerals in fluid and melt inclusions: implications for the enrichment of Be in granite-pegmatite systems. Contributions to. Mineralogy and. Petrology, 161:483-495.

Varela, M.E. 1994. Silicate-melt and fluid inclusions in rhyolitic dykes, Los Manantiales mining district, Argentina. European Journal of Mineralogy. 6, 837-54.

Vitti, C. \& Frezzotti, M.L. 2001. Transmission electron microscopy applied to fluid inclusion investigations. Lithos, 53: 125-138.

Webster, J.D. \& Duffield, W.A. 1991. Volatiles and lithophile elements in Taylor Creek Rhyolite. Constraints from glass inclusion analysis. American. Mineralogist., 76: 1628-1645.

Zhao, B., Zhao, J., Li, Z., Zhang, Z., Peng, Z. 2003. Characteristics of melt inclusions in skarn minerals from $\mathrm{Fe}, \mathrm{Cu}(\mathrm{Au})$ and $\mathrm{Au}(\mathrm{Cu})$ ore deposits in the region from Daye to Jiujiang. Science China Earth Sciences, 46: 481-497. 